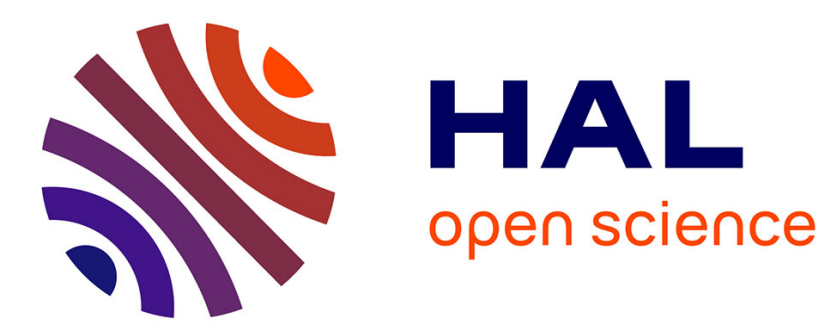

\title{
Pareto Envelopes in Simple Polygons
}

Victor Chepoi, Karim Nouioua, Edouard Thiel, Yann Vaxès

\section{To cite this version:}

Victor Chepoi, Karim Nouioua, Edouard Thiel, Yann Vaxès. Pareto Envelopes in Simple Polygons. International Journal of Computational Geometry and Applications, 2010, 20 (6), pp.707-721. 10.1142/S0218195910003499 . hal-01192933

\section{HAL Id: hal-01192933 \\ https://hal.science/hal-01192933}

Submitted on 3 Sep 2015

HAL is a multi-disciplinary open access archive for the deposit and dissemination of scientific research documents, whether they are published or not. The documents may come from teaching and research institutions in France or abroad, or from public or private research centers.
L'archive ouverte pluridisciplinaire HAL, est destinée au dépôt et à la diffusion de documents scientifiques de niveau recherche, publiés ou non, émanant des établissements d'enseignement et de recherche français ou étrangers, des laboratoires publics ou privés. 


\title{
Pareto envelopes in simple polygons ${ }^{1}$
}

\author{
Victor Chepoi, Karim Nouioua, Edouard Thiel, and Yann Vaxès
}

Laboratoire d'Informatique Fondamentale de Marseille,

Université de la Méditerranée, Faculté des Sciences de

Luminy, F-13288 Marseille Cedex 9, France,

\{chepoi,nouioua,thiel,vaxes\}@lif.univ-mrs.fr

\begin{abstract}
For a set $T$ of $n$ points in a metric space $(X, d)$, a point $y \in X$ is dominated by a point $x \in X$ if $d(x, t) \leq d(y, t)$ for all $t \in T$ and there exists $t^{\prime} \in T$ such that $d\left(x, t^{\prime}\right)<d\left(y, t^{\prime}\right)$. The set of nondominated points of $X$ is called the Pareto envelope of T. H. Kuhn (1973) established that in Euclidean spaces, the Pareto envelopes and the convex hulls coincide. Chalmet et al. (1981) characterized the Pareto envelopes in the rectilinear plane $\left(\mathbb{R}^{2}, d_{1}\right)$ and constructed them in $O(n \log n)$ time. In this note, we investigate the Pareto envelopes of point-sets in simple polygons $P$ endowed with geodesic $d_{2^{-}}$or $d_{1}$-metrics (i.e., Euclidean and Manhattan metrics). We show that Kuhn's characterization extends to Pareto envelopes in simple polygons with $d_{2}$-metric, while that of Chalmet et al. extends to simple rectilinear polygons with $d_{1}$-metric. These characterizations provide efficient algorithms for construction of these Pareto envelopes.
\end{abstract}

Keywords. Pareto envelope, dominance, geodesic $l_{1}$ - and $l_{2}$-distance, simple polygon, algorithm.

Corresponding author: Victor Chepoi

\footnotetext{
${ }^{1}$ An abstract of this paper appeared in: Proc. 24th European Workshop on Computational Geometry (EWCG'08), Nancy. This research was partly supported by the ANR grant BLAN06-1-138894 (projet OPTI$\mathrm{COMB})$
} 


\section{Introduction}

Convex hulls, in particular convex hulls in 2- and 3-dimensional spaces, are used in various applications and represent a basic object of investigations in computational geometry. They host such remarkable points as center, barycenter, and median as well as the optimal solutions of some NP-hard problems like the Steiner tree, the $p$-median, and the $p$-center problems. $\mathrm{H}$. Kuhn [18] noticed that $\operatorname{conv}(T)$ can be described in truly distance terms: a point $p \in \mathbb{R}^{m}$ belongs to $\operatorname{conv}(T)$ if and only if the vector of Euclidean distances of $p$ to the points of $T$ is not dominated by the distance vector of any other point of $\mathbb{R}^{m}$. Inspired by this characterization of $\operatorname{conv}(T)$, one can define analogous geometric objects by replacing the Euclidean distance $d_{2}$ by any other distance $d$ on $\mathbb{R}^{m}$, or by replacing $\mathbb{R}^{m}$ by a polygonal or a polyhedral domain endowed with an intrinsic distance. This leads to the following general concept of Pareto envelope. Given a set $T=\left\{t_{1}, \ldots, t_{n}\right\}$ of $n$ points in a metric space $(X, d)$, a point $y \in X$ is dominated by a point $x \in X$ if $d\left(x, t_{i}\right) \leq d\left(y, t_{i}\right)$ for all $t_{i} \in T$ and there exists $t_{j} \in T$ such that $d\left(x, t_{j}\right)<d\left(y, t_{j}\right)[2,23,28]$. The set of non-dominated points of $X$ is called the Pareto envelope of $T$ and is denoted by $\mathcal{P}_{d}(T)$.

Pareto envelopes have been investigated in several papers under the name of "sets of efficient points". Thisse, Ward, and Wendell [23] proved that $\mathcal{P}_{d_{2}}(T)=\operatorname{conv}(T)$ holds for all distances induced by round norms [25] (i.e., with strictly convex unit balls). The investigation of Pareto envelopes for particular polyhedral norms has been initiated by Wendell, Hurter, Lowe [28] and continued by Chalmet, Francis, Kolen [2] and Durier, Michelot [8, 9]. The main result of $[2]$ is the following nice characterization of Pareto envelopes in the Manhattan plane:

$$
\mathcal{P}_{d_{1}}(T)=\cap_{i=1}^{n}\left(\cup_{j=1}^{n} I_{d_{1}}\left(t_{i}, t_{j}\right)\right),
$$

where $I_{d_{1}}\left(t_{i}, t_{j}\right)$ is the smallest axis-parallel rectangle with diagonal $\left[t_{i}, t_{j}\right]$. This result was used in [2] to establish the correctness of an optimal $O(n \log n)$ sweeping-line algorithm for constructing $\mathcal{P}_{d_{1}}(T)$ in $\mathbb{R}^{2}$. Subsequently, Pelegrin and Fernandez [20] described an algorithm for constructing Pareto envelopes in the plane endowed with a polygonal norm. Recently, Chepoi and Nouioua [5] characterized $\mathcal{P}_{d_{1}}(T)$ in $\left(\mathbb{R}^{3}, d_{1}\right)$ and showed that the characterization of Chalmet et al. [2] holds for $\mathcal{P}_{d_{\infty}}(T)$ in $\left(\mathbb{R}^{m}, d_{\infty}\right)$. They also presented efficient algorithms for constructing $\mathcal{P}_{d_{1}}(T)$ and $\mathcal{P}_{d_{\infty}}(T)$ in $\mathbb{R}^{3}$. In several papers $[2,8,9,12,14,20,23,27,28]$, Pareto envelopes are used to reduce the search space of some optimization problems by showing that they host all or at least one optimal solution(s) of the respective problems. For example, Wendell and Hurter [27] establish this type of results for the Weber problem (the weighted version of the median problem) while Hansen, Perreur, and Thisse [15] proved a similar result for the NP-hard multifacility location problem. In [6], it was shown that Pareto envelopes in the $l_{1}$-plane contain at least one minimum Manhattan network, and this fact was used in all factor 2 approximation algorithms [6, 12, 14, 19] for the NP-hard [7] minimum Manhattan network problem. For other results in this vein, see [23].

In this note, we characterize and efficiently construct the Pareto envelopes of sets in simple polygons and simple rectilinear polygons $P$ endowed with the geodesic $d_{2}$ and $d_{1^{-}}$ distances, respectively. Distance problems for simple polygons constitute a classical subject in 

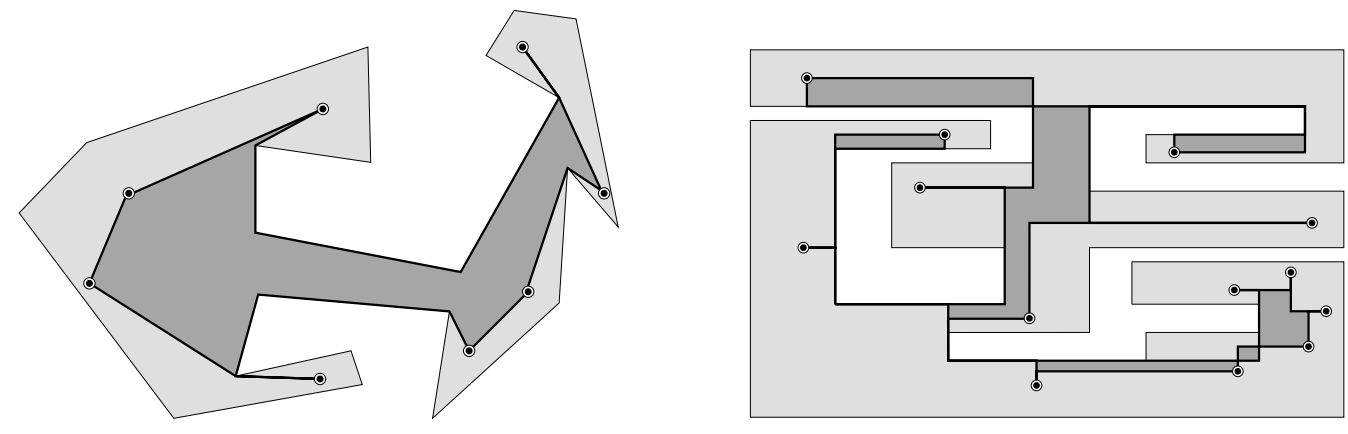

Figure 1: Examples of $\mathcal{P}_{d_{2}}(T)$ and $\mathcal{P}_{d_{1}}(T)$

computational geometry; $[11,16,21,22,24]$ is a small sample of papers devoted to this subject. We show that, like in Euclidean spaces, Pareto envelopes of finite sets in simple polygons with $d_{2}$-distance coincide with their geodesic convex hulls and therefore can be constructed using an algorithm of Toussaint [24]. On the other hand, we show that Pareto envelopes in simple rectilinear polygons can be characterized using equality (1). This characterization is used to design an efficient algorithm for constructing these envelopes.

We conclude this section with some definitions. Let $(X, d)$ be a metric space. The interval $I(x, y)$ between two points $x, y \in X$ consists of all points between $x$ and $y: I(x, y):=\{u \in$ $X: d(x, u)+d(u, y)=d(x, y)\}$. A set $M$ of $X$ is convex if $I(x, y) \subseteq M$ for all $x, y \in M$. The convex hull $\operatorname{conv}(S)$ of a set $S \subset X$ is the smallest convex set containing $S$.

\section{Simple polygons}

In this section, $P$ is a simple polygon with $m$ sides endowed with the geodesic $d_{2}$-metric. For two points $x, y \in P, \gamma(x, y)$ is the unique geodesic path inside $P$ between $x$ and $y$, and $d_{2}(x, y)$ is the length of this path. For a set of $n$ points $T \subset P$, we denote by $\operatorname{conv}(T)$ and $\mathcal{P}_{d_{2}}(T)$ the geodesic convex hull and the Pareto envelope of $T$. Since two points of a simple polygon $P$ are connected by a unique geodesic, $\left(P, d_{2}\right)$ is a metric space of global non-positive curvature, i.e., a $C A T(0)$-space [1]. $\mathrm{CAT}(0)$ spaces are characterized in several ways (in particular, by uniqueness of geodesic paths, convexity of the distance function, etc.) and have many important properties, placing them in the center of modern geometry; for results and definitions the reader can consult the book [1]. Below we will show that $\mathcal{P}_{d}(T) \subseteq \operatorname{conv}(T)$ holds for any finite subset of a $\mathrm{CAT}(0)$-space $(X, d)$ and we conjecture that in fact $\mathcal{P}_{d}(T)=\operatorname{conv}(T)$ holds.

\section{$2.1 \quad \mathcal{P}_{d_{2}}(T)=\operatorname{conv}(T)$}

We aim to establish the following result:

Proposition $1 \mathcal{P}_{d_{2}}(T)=\operatorname{conv}(T)$. Consequently, $\mathcal{P}_{d_{2}}(T)$ can be constructed in $O(m+$ $n \log m)$-time. 
The inclusion $\mathcal{P}_{d_{2}}(T) \subseteq \operatorname{conv}(T)$ follows from the following more general result which holds for all $\mathrm{CAT}(0)$ metric spaces:

Lemma 2.1 $\mathcal{P}_{d}(T) \subseteq \operatorname{conv}(T)$ for any finite set of a $C A T(0)$ metric space $(X, d)$.

Proof. Let $x \notin \operatorname{conv}(T)$. By Proposition 2.4(1) of [1] there exists a unique point $\pi(x)$ (the metric projection of $x$ ) such that $d(x, \pi(x))=\inf _{y \in \operatorname{conv}(T)} d(x, y)$. As in the case of Euclidean spaces, $\pi(x)$ can be viewed as the orthogonal projection of $x$ on $\operatorname{conv}(T)$, because by Proposition 2.4(3) the Alexandrov angle $\alpha$ at $\pi(x)$ between the geodesics $\gamma(x, \pi(x))$ and $\gamma(y, \pi(x))$ is at least $\pi / 2$ for any point $y \in \operatorname{conv}(T), y \neq \pi(x)$. By law of cosines which holds in CAT(0) spaces (page 163 of [1]), if $a=d(x, \pi(x)), b=d(y, \pi(x))$, and $c=d(x, y)$, then $c^{2} \geq a^{2}+b^{2}-2 a b \cos \alpha \geq a^{2}+b^{2}>b^{2}$ for any $y \in \operatorname{conv}(T), y \neq \pi(x)$. Hence $d(x, y)>d(\pi(x), y)$, i.e., $x$ is dominated by $\pi(x)$ yielding $x \notin \mathcal{P}_{d}(T)$. Since $x$ is an arbitrary point outside $\operatorname{conv}(T)$, we conclude that $\mathcal{P}_{d}(T) \subseteq \operatorname{conv}(T)$.

Now we show the converse inclusion $\operatorname{conv}(T) \subseteq \mathcal{P}_{d_{2}}(T)$. Pick $q \in \operatorname{conv}(T)$. If $q$ belongs to the boundary of $\operatorname{conv}(T)$, then $q$ belongs to the geodesic path $\gamma\left(t, t^{\prime}\right)$ between two vertices $t, t^{\prime}$ of $\operatorname{conv}(T)$. Since $t, t^{\prime} \in T$, if $q$ is dominated by some point $p$, then $d_{2}(p, t) \leq d_{2}(q, t)$ and $d_{2}\left(p, t^{\prime}\right) \leq d\left(q, t^{\prime}\right)$. Since $q \in \gamma\left(t, t^{\prime}\right)$, this is possible only if these inequalities hold as equalities, thus $p \in \gamma\left(t, t^{\prime}\right)$, yielding $p=q$. Thus $q \in \mathcal{P}_{d_{2}}(T)$ in this case. Now, suppose that $q$ belongs to the interior of the simple polygon $\operatorname{conv}(T)$. Suppose by way of contradiction that $q$ is dominated by some point $p^{\prime} \in P$; see Fig. 2 for an illustration. By Lemma 1 of [21] the distance function $d_{2}$ on $P$ is convex. This means that for any point $t \in T$, as $p$ varies along the geodesic $\gamma\left(p^{\prime}, q\right), d_{2}(t, p)$ is a convex function of $p$. Since $q$ belongs to the interior of $\operatorname{conv}(T)$, one can select a point $p \in \gamma\left(p^{\prime}, q\right) \cap \operatorname{conv}(T)$ which still dominates $q$ and is visible from $q$ (i.e., $[p, q] \subseteq P)$. Denote by $q^{\prime}$ the intersection of the boundary of $\operatorname{conv}(T)$ with the ray with origin $p$ which passes via the point $q$. By definition of $q^{\prime}$, we infer that $q \in\left[p, q^{\prime}\right]=\gamma\left(p, q^{\prime}\right)$. Pick any point $t \in T$. By second part of Lemma 1 of [21], $d_{2}(t, q)<\max \left\{d_{2}\left(t, q^{\prime}\right), d_{2}(t, p)\right\}$. Since $d_{2}(t, p) \leq d_{2}(t, q)$ by the choice of $p$, we obtain that $d_{2}(t, q)<d_{2}\left(t, q^{\prime}\right)$. Since this inequality holds for all points of $T$, we conclude that $q$ and $p$ both dominate the boundary point $q^{\prime}$, a contradiction with the fact that $q^{\prime} \in \mathcal{P}_{d_{2}}(T)$. This establishes the inclusion $\operatorname{conv}(T) \subseteq \mathcal{P}_{d_{2}}(T)$ and concludes the proof of Proposition 1.

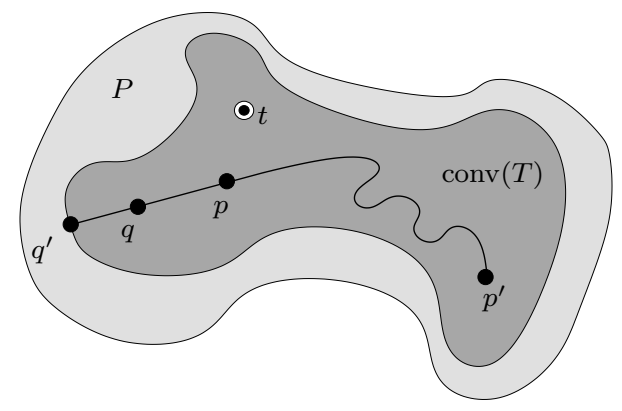

Figure 2: Illustration of the proof of the inclusion $\operatorname{conv}(T) \subseteq \mathcal{P}_{d_{2}}(T)$ 
G. Toussaint [24] presented an $O(m+n \log m)$-time algorithm for constructing the geodesic convex hull of an $n$-point set $T$ of a simple polygon $P$ with $m$ sides. Together with Proposition 1 this shows that $\mathcal{P}_{d_{2}}(T)$ can be constructed within the same running time.

\section{Simple rectilinear polygons}

In this section, $P$ is a simple rectilinear polygon (i.e., a simple polygon having all edges axisparallel) with $m$ edges endowed with the geodesic $d_{1}$-metric. A rectilinear path is a polygonal chain consisting of axis-parallel segments lying inside $P$. The length of a rectilinear path in the $d_{1}$-metric equals the sum of the length of its constituent segments. For two points $x, y \in P$, the geodesic $d_{1}$-distance $d_{1}(x, y)$ is the length of a minimum length rectilinear path (i.e., rectilinear geodesic) connecting $x$ and $y$. An axis-parallel segment $c$ is a cut segment of $P$ if it connects two edges of $P$ and lies entirely in $P$. A maximal cut is a maximal by inclusion cut segment of $P$. One basic property of the resulting metric space $\left(P, d_{1}\right)$ is that its axis-parallel cuts and the two subpolygons defined by such cuts are convex and gated [4]. A subset $M$ of a metric space $(X, d)$ is called gated [26] provided every point $v \in X$ admits a gate in $M$, i.e., a point $g(v, M) \in M$ such that $g(v, M) \in I(v, u)$ for all $u \in M$; Fig. 3 illustrates the gates of three terminals in a vertical cut $c_{v}$ of $P$.

\subsection{Characterization}

We extend the characterization of Chalmet et al. [2] to Pareto envelopes $\mathcal{P}_{d_{1}}(T)$ in simple rectilinear polygons:

Proposition $2 \mathcal{P}_{d_{1}}(T)=\cap_{i=1}^{n}\left(\cup_{j=1}^{n} I\left(t_{i}, t_{j}\right)\right)$, where $I\left(t_{i}, t_{j}\right)$ is the interval in $\left(P, d_{1}\right)$ between the terminals $t_{i}, t_{j}$ of $T$.

Proof. The direction $(\supseteq)$ of the proof is obvious: if $p$ does not belong to the Pareto envelope $\mathcal{P}_{d_{1}}(T)$, then there exists $p^{\prime} \in P$ such that $d_{1}\left(p^{\prime}, t_{j}\right) \leq d_{1}\left(p, t_{j}\right)$ for all $j \in\{1, \ldots, n\}$ and $d_{1}\left(p^{\prime}, t_{i}\right)<d_{1}\left(p, t_{i}\right)$ for some $i$. Hence, $d_{1}\left(t_{i}, p^{\prime}\right)+d_{1}\left(p^{\prime}, t_{j}\right)<d_{1}\left(t_{i}, p\right)+d_{1}\left(p, t_{j}\right)$ for all $j \in$ $\{1, \ldots, n\}$ and thus $p \notin \cup_{j=1}^{n} I\left(t_{i}, t_{j}\right)$.

To prove the converse inclusion $(\subseteq)$, let $p \in \mathcal{P}_{d_{1}}(T)$ but $p \notin \cup_{j=1}^{n} I\left(t_{i}, t_{j}\right)$ for some $t_{i}$. Let $c_{v}=\left[q^{\prime}, q^{\prime \prime}\right]$ and $c_{h}=\left[p^{\prime}, p^{\prime \prime}\right]$ be the maximal vertical and horizontal cuts which pass through the point $p$. Denote by $P_{1}, P_{2}, P_{3}$, and $P_{4}$ the subpolygons of $P$ defined by these cuts. Let $P_{1} \cap P_{3}=P_{2} \cap P_{4}=\{p\}$ and $t_{i} \in P_{1}$. Obviously, $P_{1}, \ldots, P_{4}$ are gated. Note that $p$ is the gate in $P_{1}$ of any point of $P_{3}$. As $p \notin \cup_{j=1}^{n} I\left(t_{i}, t_{j}\right)$, we conclude that $P_{3} \cap T=\emptyset$. Set $P_{j, k}:=P_{j} \cup P_{k}$, where $j, k \in\{1,2,3,4\}$ and $j \neq k$. Note that the four subpolygons $P_{j, j+1(\bmod 4)}$ are gated sets of $P$.

First, suppose that $p$ is the gate of $t_{i}$ in one of the cuts $c_{h}$ or $c_{v}$, say in $c_{h}$; see Fig. 3. Then $p$ is also the gate of $t_{i}$ in $P_{3,4}$. Indeed, the gate $q$ of $t_{i}$ in $P_{3,4}$ belongs to $c_{h}$. Since $p$ is the gate of $t_{i}$ in $c_{h}$, we have $p \in I\left(t_{i}, q\right)$, hence $p=q$. From the choice of $p$ and $t_{i}$ we conclude that $P_{3,4} \cap T=\emptyset$. Let $g_{1}, \ldots, g_{n}$ be the gates of the points $t_{1}, \ldots, t_{n}$ of $T$ in $c_{v}$. First, assume that these gates are all different from $p$. Then all $g_{1}, \ldots, g_{n}$ belong to the segment 


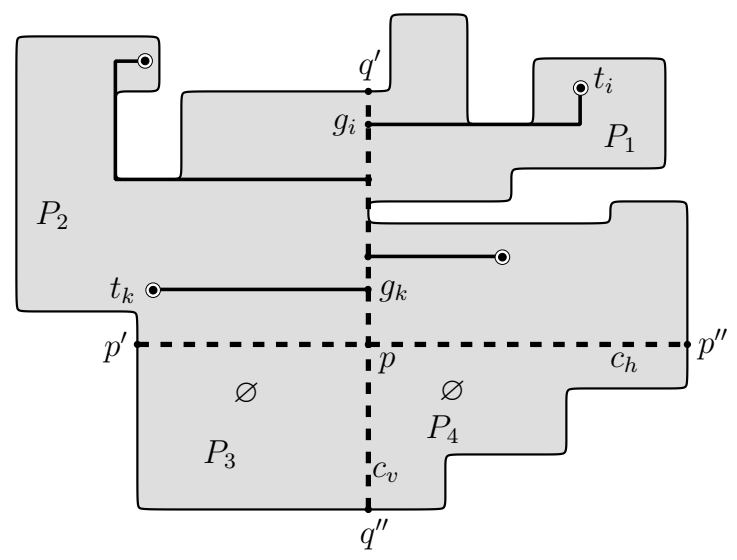

Figure 3: First case in the proof of Proposition 2

$\left[q^{\prime}, p\right] \subset c_{v}$ which separates $P_{1}$ and $P_{2}$. Let $g_{k} \in\left\{g_{1}, \ldots, g_{n}\right\}$ be the closest gate to point $p$. Then $g_{k} \in I\left(p, g_{j}\right)$ and, since $g_{j} \in I\left(p, t_{j}\right)$, we infer that $g_{k} \in \cap_{j=1}^{n} I\left(p, t_{j}\right)$, thus $g_{k}$ dominates $p$, yielding a contradiction with $p \in \mathcal{P}_{d_{1}}(T)$.

Now assume that $p$ is the gate of some point $t_{j} \neq t_{i}$ in $c_{v}$. If $t_{j} \in P_{2}$, then $p$ is the gate of $t_{j}$ in $P_{1,4}$, contrary to $p \notin I\left(t_{i}, t_{j}\right)$. Thus $t_{j} \in P_{1}$. Let $u$ and $w$ be the gates of $t_{i}$ and $t_{j}$ in $c_{v}$ and $c_{h}$, respectively. Pick some rectilinear geodesics $\gamma\left(t_{i}, u\right), \gamma\left(t_{j}, w\right)$, and $\gamma\left(t_{i}, t_{j}\right)$ between the pairs $t_{i}, u ; t_{j}, w$, and $t_{i}, t_{j}$, respectively; for an illustration, see Fig. 4 (a). Since $p \notin I\left(t_{i}, t_{j}\right)$, the geodesic $\gamma\left(t_{i}, t_{j}\right)$ cannot share common points with both segments $[u, p]$ and $[p, w]$. Let $\gamma\left(t_{i}, t_{j}\right) \cap[u, p]=\emptyset$. Let $u^{\prime}$ be a closest to $u$ point of the intersection $\gamma\left(t_{i}, u\right) \cap \gamma\left(t_{i}, t_{j}\right)$. Necessarily $u^{\prime} \neq u$. Let $w^{\prime}$ be a closest to $w$ point of the intersection $\gamma\left(t_{j}, w\right) \cap \gamma\left(t_{i}, t_{j}\right)$. Since $P$ is a simple polygon, the region of the plane bounded by $[u, p],[p, w]$, the part of $\gamma\left(t_{i}, u\right)$ between $u, u^{\prime}$, the part of $\gamma\left(t_{i}, t_{j}\right)$ between $u^{\prime}, w^{\prime}$, and the part of $\gamma\left(t_{j}, w\right)$ between $w^{\prime}, w$, is contained in $P$. Let $\left[u^{\prime \prime}, u\right]$ be the last link in the subpath of $\gamma\left(t_{i}, u\right)$ between $u^{\prime}$ and $u$. Then for some $\delta>0$, the segment $\left[v^{\prime}, v^{\prime \prime}\right]$ belongs to $P$, where $v^{\prime} \in\left[u^{\prime \prime}, u\right], v^{\prime \prime} \in[p, w]$ and $d\left(u, v^{\prime}\right)=d\left(p, v^{\prime \prime}\right)=\delta$. This contradicts that $p$ is the gate of $t_{i}$ in $c_{h}$.

Now, suppose that the gates $q$ and $z$ of $t_{i}$ in $c_{v}$ and $c_{h}$ are different from $p$. Let $u$ be the furthest from $t_{i}$ point of $I\left(t_{i}, q\right) \cap I\left(t_{i}, z\right)$. Pick the rectilinear geodesics $\gamma(u, q)$ and $\gamma(u, z)$ between $u, q$ and $u, z$. Let $\left[q^{\prime}, q\right]$ and $\left[z^{\prime}, z\right]$ be the last links of these paths. Let $q^{\prime \prime}$ be the point of $c_{h}$ with the same $x$-coordinate as $q^{\prime}$. Let $z^{\prime \prime}$ be the point of $c_{v}$ with the same $y$-coordinate as $z^{\prime}$; for an illustration, see Fig. 4 (b). Since $P$ is a simple polygon, the region between $[q, p],[z, p]$ and $\gamma(u, q), \gamma(u, z)$ belongs to $P$. Moreover, since $q, z \in I\left(p, t_{i}\right)$, and $I\left(p, t_{i}\right)$ is convex, this region necessarily belongs to $I\left(p, t_{i}\right)$. In particular, both rectangles $R^{\prime}=\left[q^{\prime}, q, p, q^{\prime \prime}\right]$ and $R^{\prime \prime}=\left[z^{\prime}, z, p, z^{\prime \prime}\right]$ belong to $I\left(p, t_{i}\right)$. As we already stated, all points of $T$ are outside $P_{3}$. Consider the gates in $c_{v}$ of the terminals of $T \cap P_{2}$ and let $g_{v}$ be the closest to $p$ such gate. Analogously, consider the gates in $c_{h}$ of the terminals of $T \cap P_{4}$ and let $g_{h}$ be the closest to $p$ such gate. Since $p \notin \cup_{j=1}^{n} I\left(t_{i}, t_{j}\right)$, we conclude that $g_{v}$ and $g_{h}$ are different from $p$. Let $0<\delta<\min \left\{d\left(p, g_{v}\right), d\left(p, g_{h}\right), d\left(z^{\prime}, z\right), d\left(q^{\prime}, q\right)\right\}$. Consider a point $p^{\prime} \in R^{\prime} \cap R^{\prime \prime}$ whose 


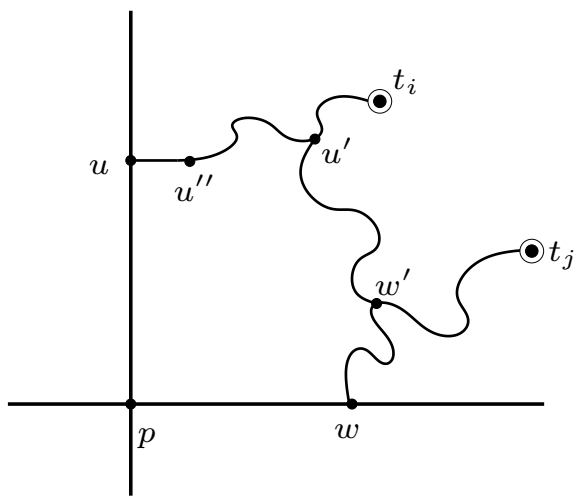

(a)

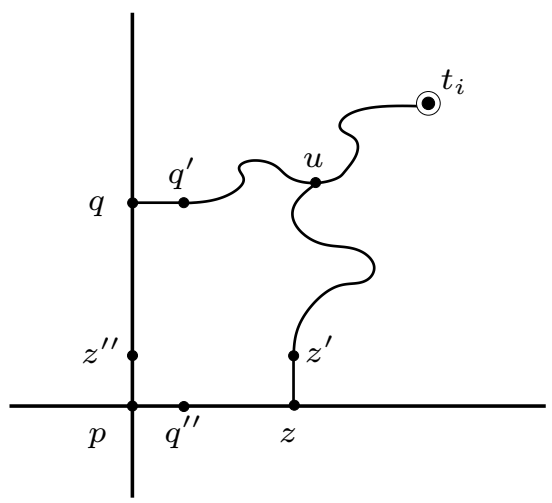

(b)

Figure 4: Second case in the proof of Proposition 2

coordinates differ by $\delta$ from those of $p$. Since $p^{\prime} \in I\left(p, t_{i}\right)$, we obtain that $d\left(p^{\prime}, t_{i}\right)=d\left(p, t_{i}\right)-2 \delta$. For any other $t_{j}$ we have $d\left(p^{\prime}, t_{j}\right) \leq d\left(p, t_{j}\right)$. This contradicts that $p \in \mathcal{P}_{d_{1}}(T)$.

A subset $S$ of $P$ is ortho-convex [13] if the intersection of $S$ with any axis-parallel cut of $P$ is connected.

Lemma $3.1 \mathcal{P}_{d_{1}}(T)$ is a closed ortho-convex set of $P$.

Proof. Proposition 2 shows that $\mathcal{P}_{d_{1}}(T)$ is closed. Since the intersection preserves orthoconvexity, by Proposition 2 it suffices to show that for any point $t_{i} \in T$ the union $\cup_{j=1}^{n} I\left(t_{i}, t_{j}\right)$ is orthogonally convex. Pick any horizontal or vertical cut $c$ of $P$. If for some point $t_{j} \in T$ the intersection $c^{\prime}:=c \cap I\left(t_{i}, t_{j}\right)$ is non-empty, then one can easily show that $c^{\prime}=\left[g\left(t_{i}, c\right), g\left(t_{j}, c\right)\right]$, where $g\left(t_{i}, c\right)$ and $g\left(t_{j}, c\right)$ are the gates of the points $t_{i}, t_{j}$ in $c$. Since the intersection of $c$ with $\cup_{j=1}^{n} I\left(t_{i}, t_{j}\right)$ is the union of such segments $\left[g\left(t_{i}, c\right), g\left(t_{j}, c\right)\right]$ and all these segments share the point $g\left(t_{i}, c\right)$, this union is necessarily a segment as well, thus we are done.

\subsection{The algorithm}

Now, we describe the algorithm for constructing the Pareto envelope $\mathcal{P}_{d_{1}}(T)$ for a set $T$ of $n$ terminals in a simple rectilinear polygon $P$ with $m$ vertices. We start with a general description of the algorithm and then we describe each of its steps in details.

First, the polygon $P$ is decomposed into rectangles by employing only horizontal cuts which pass through the vertices of $P$. This subdivision is further refined by dividing each rectangle containing terminals with the horizontal cuts passing via terminals. Denote by $\mathcal{D}$ the resulting decomposition of $P$ into rectangles. Each rectangle $R$ of $\mathcal{D}$ is a gated set. At the next step, using two sweepings of $\mathcal{D}$, for each rectangle $R$ of $\mathcal{D}$, we compute the leftmost and the rightmost gates on each of its horizontal sides of the terminals located in the subpolygons defined by these sides. We denote this quadruple of extremal gates by $Q_{R}$. Then, for each 
$R \in \mathcal{R}$, we compute the Pareto envelope $\mathcal{P}_{d_{1}}\left(Q_{R}\right)$ of the quadruple $Q_{R}$. It consists of a box $B_{R}$ and two horizontal segments; these segments do not necessarily belong to the final Pareto envelope of $T$. On the other hand, we prove that $B_{R}$ minus its horizontal sides is exactly the intersection of $\mathcal{P}_{d_{1}}(T)$ with the rectangle $R$ minus its horizontal sides. To complete the set $\cup_{R \in \mathcal{D}} B_{R}$ to the full Pareto envelope $\mathcal{P}_{d_{1}}(T)$, for each horizontal cut $c$ of $\mathcal{D}$, we have to compute its intersection with $\mathcal{P}_{d_{1}}(T)$. We show that $\mathcal{P}_{d_{1}}(T) \cap c$ coincides with the smallest segment $s_{c} \subseteq c$ spanned by the terminals and/or the horizontal sides of all boxes $B_{R}$ located on $c$, and therefore can be easily computed. As a result, the algorithm returns as $\mathcal{P}_{d_{1}}(T)$ the union of all boxes $B_{R}$ and segments $s_{c}$ for all rectangles $R$ and horizontal cuts $c$ of the subdivision $\mathcal{D}$.

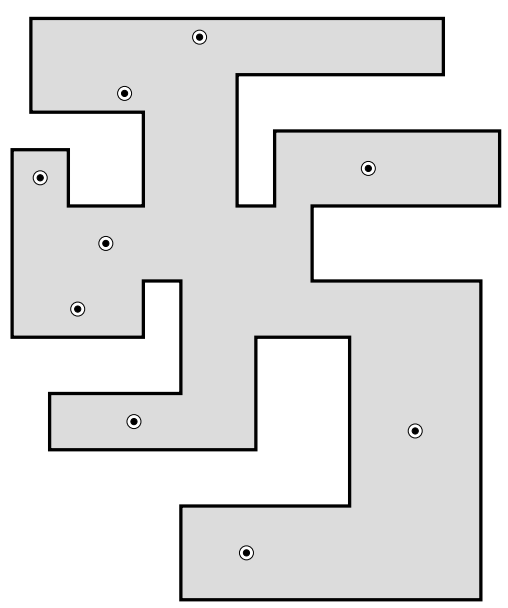

(a)

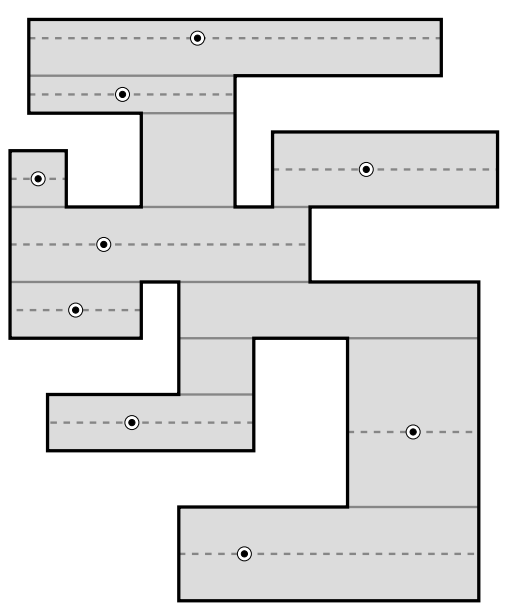

(b)

Figure 5: (a) A set $T$ of terminals in a polygon $P$. (b) The decomposition $\mathcal{D}$ of $P$ into rectangles computed during step 1.

Step 1: Computing a subdivision $\mathcal{D}$ of $P$ into rectangles. At the first step of the algorithm, the polygon $P$ is decomposed into rectangles by employing only horizontal cuts which pass through the vertices of $P$. The algorithm uses Chazelle's algorithm for computing all vertex-edge visible pairs of a simple polygon [3] and the optimal point location methods $[10,17]$. Using Chazelle's algorithm, we derive in $O(m)$ time a decomposition of the polygon $P$ into rectangles, employing only horizontal cuts which pass through the vertices of $P$. Using the optimal point location methods $[10,17]$ we compute in total $O(n \log m)$ time which rectangles of the decomposition contain the terminals (notice that the induced subdivision is monotone, whence the point location structure can be built in linear time). At the next step, we sort by $y$-coordinate all terminals from each rectangle. With these sorted lists, we refine the initial subdivision by dividing each rectangle containing terminals with the horizontal cuts passing via terminals (see Fig. 5). The dual graph of this decomposition $\mathcal{D}$ is a tree $\mathcal{T}$ : the nodes of the tree are the rectangles of $\mathcal{D}$, and two nodes in $\mathcal{T}$ are adjacent if and only if the corresponding rectangles are bounded by a common cut; for an illustration, see Fig. 6 (a). 
We suppose that $\mathcal{T}$ is rooted at some rectangle. Any cut $c$ of our subdivision divides the polygon $P$ into two subpolygons $P_{c}^{\prime}$ and $P_{c}^{\prime \prime}$ which correspond to two subtrees $\mathcal{T}_{c}^{\prime}$ and $\mathcal{T}_{c}^{\prime \prime}$ of $\mathcal{T}$. It can be easily shown that if $P_{c}^{\prime \prime} \cap T=\emptyset$ (in this case we say that $P_{c}^{\prime \prime}$ is $T$-empty), then $\mathcal{P}_{d_{1}}(T)$ is contained in $P_{c}^{\prime} \cup c$ (any point of $P_{c}^{\prime \prime}$ is dominated by its gate in $c$ ). By processing the tree $\mathcal{T}$, in linear time we can remove all $T$-empty subpolygons and their corresponding subtrees. We will denote the resulting polygon, subdivision, and tree also by $P, \mathcal{D}$, and $\mathcal{T}$. The resulting decomposition $\mathcal{D}$ and its tree $\mathcal{T}$ can be constructed in time $O(m+n(\log n+\log m))$. If all terminals are vertices of $P$, then we avoid the application of point location methods and ranking of terminals, requiring only $O(n+m)$ time.

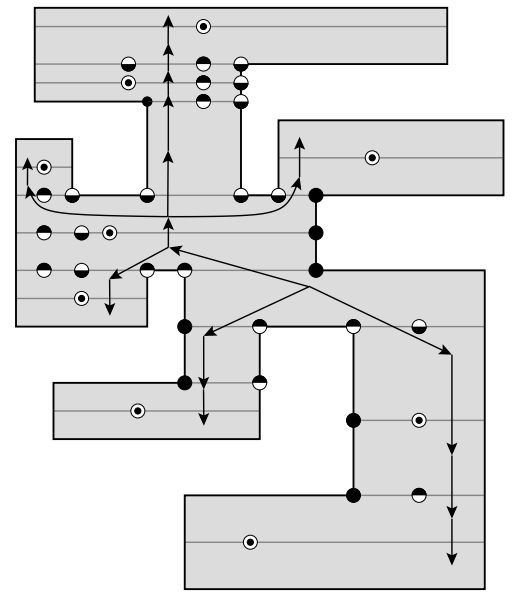

(a)

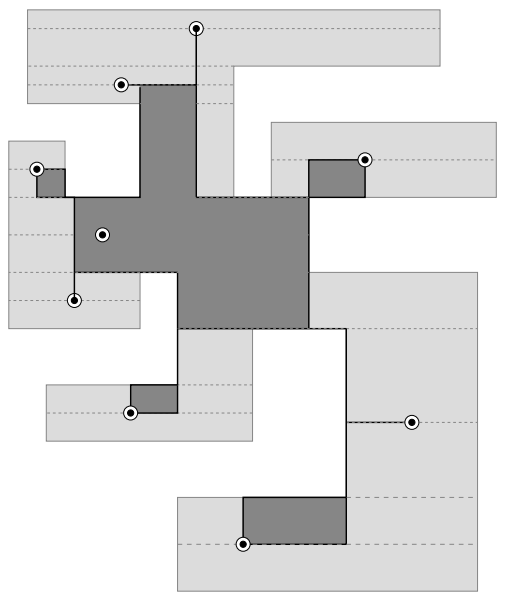

(b)

Figure 6: (a) The tree $\mathcal{T}$ and the quadruples $Q_{R}$ of extremal gates for each rectangle $R$ of the decomposition $\mathcal{D}$. (b) The Pareto envelope $\mathcal{P}_{d_{1}}(T)$.

Step 2: Computing the quadruples $Q_{R}$ of extremal gates. Given a non-root rectangle $R$ of $\mathcal{D}$, we denote by $e_{R}^{\prime}$ and $e_{R}^{\prime \prime}$ the horizontal sides of $R$, so that $e_{R}^{\prime}$ separates $R$ from the root of $\mathcal{T}$. The set of gates of all terminals in $R$ can be partitioned into the subset $G_{R}^{\prime}$ of gates located on $e_{R}^{\prime}$ and the subset $G_{R}^{\prime \prime}$ of gates located on $e_{R}^{\prime \prime}$. Let $g_{l}^{\prime}(R), g_{r}^{\prime}(R)$ be the leftmost and the rightmost points from $G_{R}^{\prime}$ and let $g_{l}^{\prime \prime}(R), g_{r}^{\prime \prime}(R)$ be the leftmost and the rightmost points from $G_{R}^{\prime \prime}$, see Fig. 6 (a). We will show now how to compute the four extremal gates $g_{l}^{\prime}(R), g_{r}^{\prime}(R), g_{l}^{\prime \prime}(R)$ and $g_{r}^{\prime \prime}(R)$ for all rectangles $R \in \mathcal{D}$ in total linear time.

In the first stage, we consider only the gates of terminals that belong to $R$ or to rectangles that are descendants of $R$ in $\mathcal{T}$ (called lower terminals) and, in the second stage, the gates of the remaining terminals (called upper terminals). At the beginning of the procedure, for each $R \in \mathcal{D}$, we assign to $g_{l}^{\prime}(R), g_{r}^{\prime}(R), g_{l}^{\prime \prime}(R), g_{r}^{\prime \prime}(R)$ the leftmost or the rightmost terminal of $e_{R}^{\prime}$ and $e_{R}^{\prime \prime}$ (or a conventional null value if such terminals do not exist). This step requires $O(n+m)$ total time. The first stage is implemented by applying the following simple upward traversal of $\mathcal{T}$. If $R$ is a leaf of $\mathcal{T}$, then $g_{l}^{\prime}(R), g_{r}^{\prime}(R), g_{l}^{\prime \prime}(R), g_{r}^{\prime \prime}(R)$ have been defined at the initialization step. Now suppose that $R$ is an inner node of $\mathcal{T}$. For each child rectangle $R^{\prime}$ of 
$R$ we already computed the leftmost and the rightmost gates of all its lower terminals. Then we compute the gates in $R$ of these four points of $R^{\prime}$ (since $R$ and $R^{\prime}$ are adjacent rectangles this requires constant time). Each time we compute a gate $g$ in $R$ (suppose it lies on $e_{R}^{\prime}$, the case of $e_{R}^{\prime \prime}$ is identical) such that either (i) $g_{l}^{\prime}(R)$ and $g_{r}^{\prime}(R)$ are null, (ii) $g$ is to the left of current $g_{l}^{\prime}(R)$ or (iii) $g$ is to right of current $g_{r}^{\prime}(R)$, we update the value of $g_{l}^{\prime}(R)$ and $g_{r}^{\prime}(R)$ accordingly.

The second stage for upper terminals is analogous, except that (i) the tree $\mathcal{T}$ is traversed in a downward way and (ii) an extremal gate of the parent rectangle $R^{\prime}$ of $R$ is taken into account for computing the extremal gates in $R$ only if it is the gate of an upper terminal (with respect to $R$ ). Since, by construction of $\mathcal{D}$, any point of $P$ belongs to at most two rectangles of $\mathcal{D}$, a gate in $R^{\prime}$ is defined by an upper terminal with respect to $R$ if and only if it does not belong to $R$. If $R$ is the root of $\mathcal{T}$, then there are no upper terminals and the gates $g_{l}^{\prime}(R)$, $g_{r}^{\prime}(R), g_{l}^{\prime \prime}(R), g_{r}^{\prime \prime}(R)$ have been already correctly computed in Phase 1 . Now suppose that $R$ is an inner node of $\mathcal{T}$ and that the extremal gates in the parent rectangle $R^{\prime}$ of $R$ have been already computed. Then among such gates we select those which do not belong to $R$ (as we noticed above, these gates are defined by upper terminals with respect to $R$ ) and compute their gates in $R$. Each time when we find a gate $g$ in $R$ (it lies on $e_{R}^{\prime}$ because $R^{\prime}$ is the parent of $R$ ) such that either (i) $g_{l}^{\prime}(R)$ and $g_{r}^{\prime}(R)$ are null, (ii) $g$ is to the left of current $g_{l}^{\prime}(R)$ or (iii) $g$ is to the right of current $g_{r}^{\prime}(R)$, then we update the values of $g_{l}^{\prime}(R)$ and $g_{r}^{\prime}(R)$ accordingly.

Lemma 3.2 At the end of the two stages of the algorithm, for each rectangle $R$ of $\mathcal{D}$, the quadruple $Q_{R}=\left\{g_{l}^{\prime}(R), g_{r}^{\prime}(R), g_{l}^{\prime \prime}(R), g_{r}^{\prime \prime}(R)\right\}$ of extremal gates has been correctly computed.

Proof. The correctness of the first stage is proved by induction. Pick $R \in \mathcal{D}$ and suppose that the procedure correctly computes the four extremal gates for its children. Suppose that there is a lower terminal $t$ whose gate $g_{t}$ in $R$ lies on $e_{R}^{\prime}$ to the left of $g_{l}^{\prime}(R)$. Suppose that $t$ lies in the subpolygon defined by the child rectangle $R^{\prime}$. The choice of $g_{t}$ implies that $t \notin R^{\prime}$ and therefore the gate of $t$ in $R$ coincides with the gate in $R$ of its gate in $R^{\prime}$. From the algorithm and the induction hypothesis we conclude that the gate $g_{t}^{\prime}$ of $t$ in $R^{\prime}$ cannot be the leftmost gate in $R^{\prime}$, otherwise $g_{t}^{\prime}$ will be involved in the computation of $g_{l}^{\prime}(R)$. Therefore $g_{t}^{\prime}$ lies to the right of $g_{l}^{\prime}\left(R^{\prime}\right)$. Suppose that $g_{l}^{\prime}\left(R^{\prime}\right)$ is the gate $g_{v}^{\prime}$ in $R^{\prime}$ of a terminal $v$. The procedure ensures that $g_{l}^{\prime}(R)$ coincides or lies to the left of the gate $g_{v}$ of $g_{v}^{\prime}$ in $R$. Since $g_{t}$ is to the left of $g_{l}^{\prime}(R)$, any two shortest paths between $g_{t}^{\prime}, g_{t}$ and $g_{v}^{\prime}, g_{v}$ have a common point $z$. By definition of $g_{t}$ and $g_{v}$, the points $z$ and $g_{t}$ lie on a common shortest path between $g_{t}^{\prime}$ and $g_{v}$, while $z$ and $g_{v}$ lie on a common shortest path between $g_{v}^{\prime}$ and $g_{t}$, and we conclude that $g_{t}=g_{v}$, a contradiction.

The correctness proof of the second stage is analogous. We pick a rectangle $R \in \mathcal{D}$, its parent rectangle $R^{\prime}$ in $\mathcal{T}$ and suppose that the procedure correctly computed the four extremal gates for $R^{\prime}$. Then, since every shortest path between an upper terminal (with respect to $R$ ) and any point in $R$ passes via $R^{\prime}$, the gates in $R$ of the upper terminals are exactly the gates in $R$ of their gates in $R^{\prime}$. Now, we proceed as in the proof of the first stage: we suppose that there is an upper terminal $t$ whose gate $g_{t}$ in $R$ lies on $e_{R}^{\prime}$ to the left of $g_{l}^{\prime}(R)$. The gate $g_{t}^{\prime}$ of $t$ in $R^{\prime}$ cannot be the leftmost gate in $R^{\prime}$, otherwise $g_{t}^{\prime}$ will be involved in the computation of $g_{l}^{\prime}(R)$. Therefore $g_{t}^{\prime}$ lies to the right of $g_{l}^{\prime}\left(R^{\prime}\right)$. Suppose that $g_{l}^{\prime}\left(R^{\prime}\right)$ is the gate $g_{v}^{\prime}$ in $R^{\prime}$ of a 

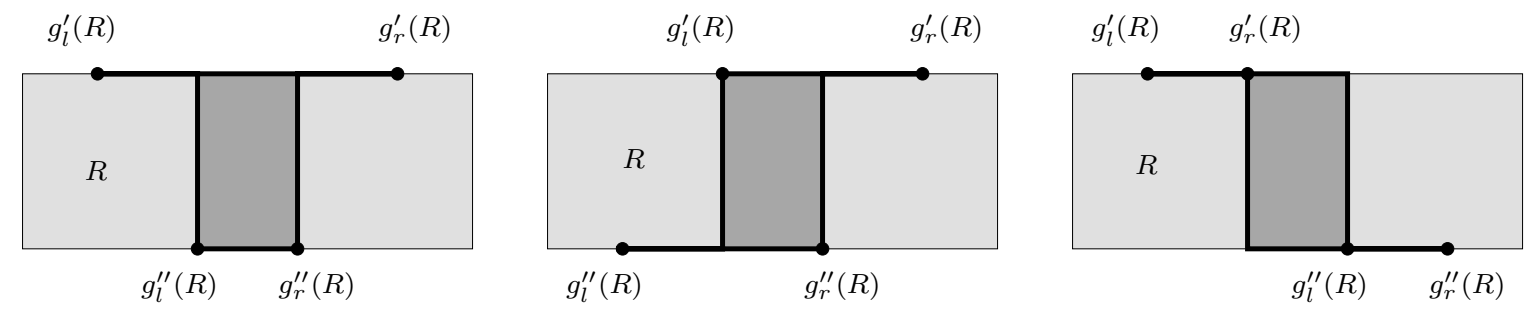

Figure 7: The three shapes of $\mathcal{P}_{d_{1}}\left(Q_{R}\right)$

terminal $v$. The procedure ensures that $g_{l}^{\prime}(R)$ coincides or lies to the left of the gate $g_{v}$ of $g_{v}^{\prime}$ in $R$. Since $g_{t}$ is to the left of $g_{l}^{\prime}(R)$, any two shortest paths between $g_{t}^{\prime}, g_{t}$ and $g_{v}^{\prime}, g_{v}$ have a common point $z$, leading to a similar contradiction as in the first case.

It can be easily seen that the total complexity of Step 2 is linear in the number of terminals and rectangles, thus $O(n+m)$.

Step 3: Computing the Pareto envelopes $\mathcal{P}_{d_{1}}\left(Q_{R}\right)$ and the boxes $B_{R}$. For each rectangle $R \in \mathcal{D}$, given the quadruple of gates $Q_{R}=\left\{g_{l}^{\prime}(R), g_{r}^{\prime}(R), g_{l}^{\prime \prime}(R), g_{r}^{\prime \prime}(R)\right\}$, at the next step we compute in constant time (and therefore in total $O(n+m)$ time) the Pareto envelope $\mathcal{P}_{d_{1}}\left(Q_{R}\right)$ of $Q_{R}$. It consists of a box $B_{R}$ having its horizontal sides on the sides of $R$ and two horizontal segments which are incident either to two points of the quadruple lying on the same horizontal side of $R$ or to two opposite points lying on different horizontal sides of $R$ (one or both these segments can be degenerated); see Fig. 5 for the three occurring cases. In general, these segments do not necessarily belong to the final Pareto envelope $\mathcal{P}_{d_{1}}(T)$. On the other hand, as we will show now, $B_{R}$ minus its horizontal sides is exactly the set $\mathcal{P}_{d_{1}}(T) \cap R^{0}$, where $R^{0}:=R \backslash\left(e_{R}^{\prime} \cup e_{R}^{\prime \prime}\right)$ (clearly, the horizontal sides of $B_{R}$ belong to $\mathcal{P}_{d_{1}}(T)$ as well because $\mathcal{P}_{d_{1}}(T)$ is closed).

Lemma $3.3 \mathcal{P}_{d_{1}}(T) \cap R^{0}=B_{R} \cap R^{0}$.

Proof. Pick any point $p \in B_{R} \cap R^{0}$. To show that $p \in \mathcal{P}_{d_{1}}(T)$, by Proposition 2 it suffices to show that $p \in \cup_{j=1}^{n} I\left(t_{i}, t_{j}\right)$ for any $t_{i} \in T$. Suppose that the gate $g$ of $t_{i}$ in $R$ belongs to $e_{R}^{\prime}$. Consider the points $g_{l}^{\prime \prime}(R)$ and $g_{r}^{\prime \prime}(R)$ and suppose that they are the gates of the terminals $t_{j}$ and $t_{k}$, respectively. First suppose that the vertical projection $p^{\prime}$ of $p$ on $e_{R}^{\prime \prime}$ belongs to the segment $\left[g_{l}^{\prime \prime}(R), g_{r}^{\prime \prime}(R)\right]$. Now if the projection of $g$ on $e_{R}^{\prime \prime}$ is to the left of that of $p$, then $p \in I\left(t_{i}, t_{k}\right)$, otherwise $p \in I\left(t_{i}, t_{j}\right)$. On the other hand, if $p^{\prime} \notin\left[g_{l}^{\prime \prime}(R), g_{r}^{\prime \prime}(R)\right]$, then we can suppose without loss of generality that $p^{\prime}$ is to the right of $g_{r}^{\prime \prime}(R)$. Since $p \in B_{R}$, the vertical projection of $p$ on $e_{R}^{\prime}$ is to the left of $g_{l}^{\prime}(R)$. Then again $p \in I\left(t_{i}, t_{j}\right)$. This establishes that $B_{R} \cap R^{0} \subseteq \mathcal{P}_{d_{1}}(T)$. Conversely, we assert that any point $q \in R^{0} \backslash B_{R}$ does not belong to $\mathcal{P}_{d_{1}}(T)$. Suppose without loss of generality that $q$ is located to the left of $B_{R}$ and that $g_{l}^{\prime \prime}(R)$ is a corner of $B_{R}$. Let $t_{i}$ be the terminal defining the gate $g_{r}^{\prime}(R)$. Then it can be easily seen that $q \notin I\left(t_{i}, t_{j}\right)$ for any other terminal $t_{j}$. Indeed, this is evidently true if the gate of $t_{j}$ in $R$ belongs to $e_{R}^{\prime}$. On the other hand, if this gate belongs to $e_{R}^{\prime \prime}$, then this gate is to the right of $g_{l}^{\prime \prime}$, thus to the right of $q$, showing that $q \notin I\left(t_{i}, t_{j}\right)$. This concludes the proof. 


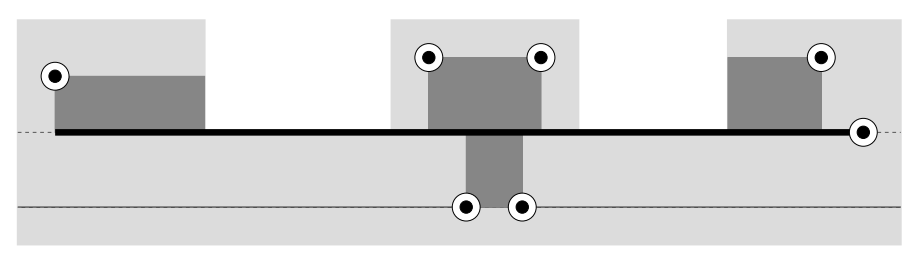

Figure 8: Computing the segments $s_{c}=\mathcal{P}_{d_{1}}(T) \cap c$

Step 4: Computing the segments $s_{c}=\mathcal{P}_{d_{1}}(T) \cap c$. For any horizontal cut $c$, we compute the smallest segment $s_{c} \subseteq c$ spanned by the terminals and/or the horizontal sides of all boxes $B_{R}$ located on $c$; for an illustration, see Fig. 8 . With the rectangles $B_{R}$ at hand, the computation of the segments $s_{c}$ can be easily done in total $O(n+m)$ time. Now, we will show that the segments $s_{c}$ represent the completion of $\cup_{R \in \mathcal{D}} B_{R}$ to $\mathcal{P}_{d_{1}}(T)$ :

Lemma 3.4 $\mathcal{P}_{d_{1}}(T) \cap c=s_{c}$.

Proof. Since by Lemma 3.1 the set $\mathcal{P}_{d_{1}}(T)$ is closed and ortho-convex, the definition of $s_{c}$ implies that $s_{c} \subset \mathcal{P}_{d_{1}}(T)$. Let $s_{c}=[u, v]$, where $u$ is to the left of $v$. Pick any point $q \in c$ to the left of $u$. Suppose by way of contradiction that $q \in \mathcal{P}_{d_{1}}(T)$. By Proposition 2, for any $t_{i} \in T$ there exists $t_{j} \in T$ such that $q \in I\left(t_{i}, t_{j}\right)$. This implies that $q$ is comprised between the gates of the points $t_{i}$ and $t_{j}$ on $c$, in particular, one of these gates coincides with $q$ or is to the left of $q$. Let $t$ be the terminal having the leftmost gate $g_{t}$ in $c$. Suppose that $I\left(t, g_{t}\right)$ crosses the rectangle $R$ having one horizontal side on $c$, say $e_{R}^{\prime}$. Then necessarily the gate of $t$ in $R$ is the point $g_{l}^{\prime \prime}(R)$. The definition of $s_{c}$ and the choice of $q$ implies that $g_{l}^{\prime \prime}(R)$ is not a corner of the box $B_{R}$. Let $u^{\prime}$ be the corner of $B_{R}$ incident to $g_{l}^{\prime \prime}(R)$ in the Pareto envelope $\mathcal{P}_{d_{1}}\left(Q_{R}\right)$. From the definition of $u$ we infer that $u \in e_{R}^{\prime}$ and that $u$ is a gate of some terminal. Then one can easily see that $\left[u^{\prime}, u\right]$ is a vertical side of $B_{R}$. By the definition of the four gates of $Q_{R}$ and the form of $\mathcal{P}_{d_{1}}\left(Q_{R}\right)$ we deduce that either $u^{\prime}=g_{r}^{\prime \prime}(R)$ or $u=g_{l}^{\prime}(R)$. In the first case all gates of $G_{R}^{\prime}$ are located to the right of $u$. Thus, if $t^{\prime}$ is the terminal defining the gate $g_{r}^{\prime \prime}(R)$, then $q$ does not belong to the interval between $t^{\prime}$ and any terminal $t_{j}$ having a gate in $G_{R}^{\prime}$. Since $q$ does not belong to $I\left(t^{\prime}, t_{j}\right)$ for any terminal having a gate in $G_{R}^{\prime \prime}$, we infer that $q \notin \cup_{j=1}^{n} I\left(t^{\prime}, t_{j}\right)$. On the other hand, if $u=g_{l}^{\prime}(R)$, then necessarily $g_{r}^{\prime \prime}(R)$ exists and is located to the right of $u^{\prime}$. By definition all gates from $G_{R}^{\prime}$ are located between $u$ and $g_{r}^{\prime}(R)$, thus to the right of $q$. This implies again that $q \notin \cup_{j=1}^{n} I\left(t^{\prime}, t_{j}\right)$, where $t^{\prime}$ is the terminal defining the gate $g_{r}^{\prime \prime}(R)$. Hence, in both cases Proposition 2 yields $q \notin \mathcal{P}_{d_{1}}(T)$, establishing our assertion.

In view of Lemmata 3.3 and 3.4, it suffices to output as $\mathcal{P}_{d_{1}}(T)$ the union of boxes $B_{R}$ for all rectangles $R$ and segments $s_{c}$ for all horizontal cuts $c$ of the subdivision $\mathcal{D}$ (Step $\mathbf{5}$ of the algorithm). Summarizing the results of this section, we obtain the following algorithm and theorem for computing $\mathcal{P}_{d_{1}}(T)$ : 


\section{Algorithm Pareto-RP $(P, T)$}

Input: A simple rectilinear polygon $P$ and a set $T \subset P$ of $n$ terminals.

Output: The Pareto envelope $\mathcal{P}_{d_{1}}(T)$.

1. Compute the subdivision $\mathcal{D}$ into rectangles of the polygon $P$ and refine $\mathcal{D}$ by dividing each rectangle containing terminals with the horizontal cuts passing via these terminals.

2. For each rectangle $R$ of $\mathcal{D}$, compute the quadruple of extremal gates $Q_{R}=\left\{g_{l}^{\prime}(R), g_{r}^{\prime}(R), g_{l}^{\prime \prime}(R), g_{r}^{\prime \prime}(R)\right\}$.

3. For each rectangle $R$ of $\mathcal{D}$, compute the Pareto envelope $\mathcal{P}_{d_{1}}\left(Q_{R}\right)$ and the box $B_{R}$ spanned by $R^{0} \cap \mathcal{P}_{d_{1}}\left(Q_{R}\right)$.

4. For each horizontal cut $c$ of $\mathcal{D}$, compute the segment $s_{c}$ spanned by the terminals and/or the horizontal sides of all boxes $B_{R}$ located on $c$.

5. Output the union of all boxes $B_{R}$ and segments $s_{c}$ for all rectangles $R$ and horizontal cuts $c$ of the subdivision $\mathcal{D}$.

Theorem 3.5 The Pareto envelope of $n$ terminals located in a simple rectilinear polygon $P$ with $m$ edges can be constructed in time $O(n+m(\log n+\log m))(O(n+m)$ if all terminals are vertices of $P$ ).

Acknowledgments. We would like to acknowledge the two anonymous referees for a careful reading of the first version of the manuscript and helpful suggestions.

\section{References}

[1] M. Bridson and A. Haefliger, Metric Spaces of Non-Positive Curvature (Springer, Berlin, 1999).

[2] G. Chalmet, L. Francis, and A. Kolen, Finding efficient solutions for rectilinear distance location problems efficiently, Europ. J. Oper. Res. 6 (1981) 117-124.

[3] B. Chazelle, Triangulating a simple polygon in linear time, Discr. Comput. Geom. 6 (1991) 485-524.

[4] V. Chepoi and F.F. Dragan, Computing a median point of a simple rectilinear polygon, Inf. Process. Lett. 49 (1994) 281-285.

[5] V. Chepoi and K. Nouioua, Pareto envelopes in $\mathbb{R}^{3}$ under $l_{1}$ and $l_{\infty}$-distance functions, in: Symposium on Computational Geometry 2007, pp. 284-293.

[6] V. Chepoi, K. Nouioua, and Y. Vaxès, A rounding algorithm for approximating minimum Manhattan networks, Theoret. Comput. Sci. 390 (2008) 56-69 (extended abstract appeared in: APPROX-RANDOM'2005, pp. 40-51). 
[7] F.Y.L. Chin, Z. Guo, and H. Sun, Minimum Manhattan network is NP-complete, in: Symposium on Computational Geometry, 2009, pp. 393-402.

[8] R. Durier, On Pareto optima, the Fermat-Weber problem, and polyhedral gauges, Math. Prog. 47 (1990) 65-79.

[9] R. Durier and C. Michelot, Sets of efficient points in normed space, J. Math. Analys. Appl. 117 (1986) 506-528.

[10] H. Edelsbrunner, L.J. Guibas, and J. Stolfi, Optimal point location in a monotone subdivision, SIAM J. Comput. 15 (1985) 317-340.

[11] L.J. Guibas, J. Hershberger, D. Leven, M. Sharir, and R. E. Tarjan, Linear-time algorithms for visibility and shortest path problems inside triangulated simple polygons, Algorithmica 2 (1987) 209-233.

[12] Z. Guo, H. Sun, and H. Zhu, Greedy Construction of 2-approximation minimum Manhattan network, in: Proc. 19th Int. Symp. Alg. and Comput. (ISAAC'2008) Springer LNCS 5369, 2008, pp. 4-15.

[13] E. Fink and D. Wood, Restricted-Orientation Convexity (Springer-Verlag, Berlin, 2004).

[14] B. Fuchs and A. Schulze, A fast 2-approximation of minimum Manhattan networks, ZAIK-Zentrum für Angewandte Informatik, Universität zu Köln, Preprint zaik2009-590.

[15] P. Hansen, T. Perreur, and J.F. Thisse, Location theory, dominance and convexity: some further results, Oper. Res. 28 (1980) 1241-1250.

[16] J. Hershberger and S. Suri, Matrix searching with the shortest-path metric, SIAM J. Comput. 26 (1997) 1612-1634.

[17] D.G. Kirkpatrick, Optimal search in planar subdivisions, SIAM J. Comput. 12 (1983) $28-35$.

[18] H.W. Kuhn, A note on Fermat's problem, Math. Prog. 4 (1973) 98-107.

[19] K. Nouioua, Enveloppes de Pareto et Réseaux de Manhattan: caractérisations et algorithmes, Thèse de Doctorat en Informatique, Université de la Méditerranée, 2005.

[20] B. Pelegrin and F.R. Fernandez, Determination of efficient points in multiple-objective location problems, Nav. Res. Logistics 35 (1988) 697-705.

[21] R. Pollack, M. Sharir, and G. Rote, Computing the geodesic center of a simple polygon, Discr. Comput. Geom. 4 (1989) 611-626.

[22] S. Suri, Computing geodesic furthest neighbors in simple polygons, J. Comput. Syst. Sci. 39 (1989) 220-235. 
[23] J.F. Thisse, J.E. Ward, and R.E. Wendell, Some properties of location problems with block and round norms, Oper. Res. 32 (1984) 1309-1327.

[24] G.T. Toussaint, Computing geodesic properties inside a simple polygon, Revue d'Intelligence Artificielle 3 (1989) 9-42.

[25] A.C. Thompson, Minkowski Geometry (Encyclopedia of Mathematics and Applications, Vol. 63, Cambridge Univertsity Press, 1996).

[26] M. van de Vel, Theory of Convex Structures (Elsevier, Amsterdam, 1993).

[27] R.E. Wendell and A.P. Hurter, Location theory, dominance, and convexity, Oper. Res. 21 (1973) 314-320.

[28] R.E. Wendell, A.P. Hurter, and T.J. Lowe, Efficient points in location theory, AIEE Trans. 9 (1973) 238-246. 